Supporting Information

\title{
Charge-Pattern Indicated Relaxation Dynamics and Glass Transition of Polymer Thin Films Studied by Atomic Force Microscopy
}

Zi-Hong Lin, ${ }^{\ddagger a}$ Di Gao, ${ }^{\ddagger a}$ Li Guan, ${ }^{* a}$ Meining Zhang, ${ }^{a}$ Xiaoyang Zhu, ${ }^{b}$ Yanlian Yang, ${ }^{b}$

Xiaohui Qiu, ${ }^{b}$ Jian-Ping Zhang ${ }^{a}$

${ }^{a}$ Department of Chemistry, Renmin University of China, Beijing 100872, P.R. China.

Email: guanl@,ruc.edu.cn.

${ }^{b}$ Key Laboratory of Standardization and Measurement for Nanotechnology (Chinese

Academy of Sciences), National Center for Nanoscience and Technology, Beijing

100190, P. R. China 
Figure S1 illustrates the fabricating procedure of PDMS Stamps. The PDMS stamp is fabricated using replica molding method by casting liquid PDMS prepolymer on a silicon mold with patterned micro-structures. The PDMS prepolymer is cured at a temperature of $348 \mathrm{~K}$ for 2 hour, and then the cured PDMS polymer is peeled off from the silicon substrate, forming a patterned PDMS stamp.

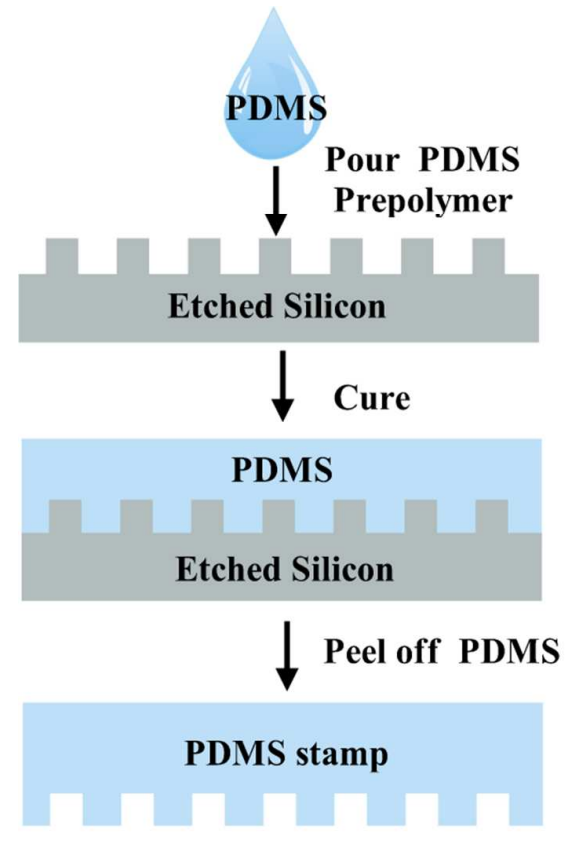

Figure S1 Schematic illustration of the procedure for fabricating PDMS stamp.

In the main text, the SPD of PMMA thin films has been discussed in detail. PMMA appears to have a stronger intermolecular force and harder to relaxation, since the hydrogen bond could be formed in the PMMA films. The same result could be observed when the polymer was changed to PS, the results are shown in Figure S2 and Figure S3. Compared with PMMA, the less interconnected polymer chain network of PS has also been chosen as it has a lower molecular weight (MW=4000) 
and weak intermolecular force. Polystyrene (PS) thin films are prepared using spin-cast, the charging processes and decay tendencies measurements are the same as that of PMMA as mentioned in the text.

Isothermal SPD curves of PS thin films $(20 \mathrm{~nm})$ under different initial surface potentials with time extension are shown in Figure S2. Moderate temperature of 323 $\mathrm{K}$ is chosen for PS according to its bulk $T_{g}$. Similar decay tendencies are observed as that of PMMA, in which a sharp decay appears in the beginning stage and a gentle decreasing followed.

The temperature dependent SPD curves of PS films with the same thickness of $20 \mathrm{~nm}$ are shown in Figure S3, in which slow decay tendencies are obtained at lower temperature and sharp drops appear when temperature increases. As mentioned in the text, the transition points are commonly considered to be related to the $\beta$ relaxation and $\alpha$-relaxation, which are respectively corresponding to the reorientation of side groups and the large scale motions of cooperative segmental mobility. It could be concluded that charges pattern indicated SPD is a feasible and effective technique to study the relaxation dynamics and $T_{g}$ properties both of PMMA and PS or other polymers thin films.

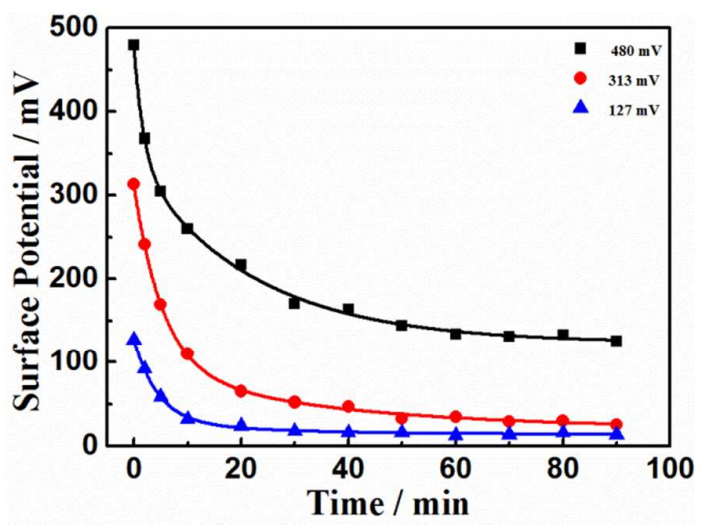


Figure S2 Isothermal SPD tendency of PS films with different initial surface potential with the same thickness of $20 \mathrm{~nm}$.

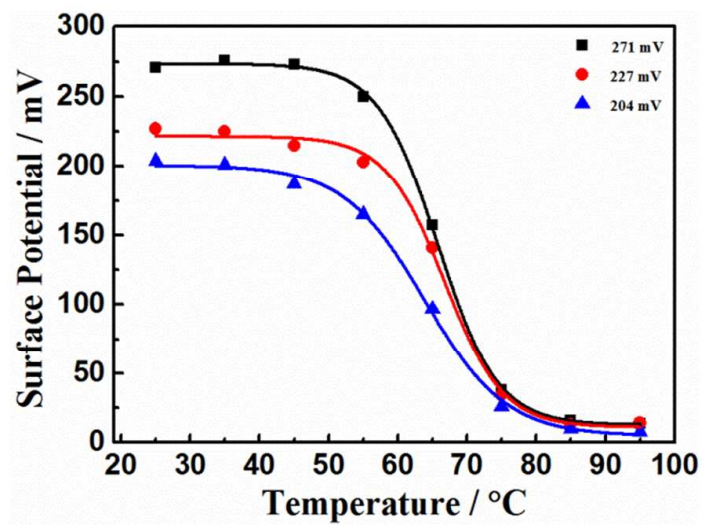

Figure S3 The temperature dependent SPD tendency of PS films with the same thickness of $20 \mathrm{~nm}$. 